\title{
Adaptive Multiresolution Denoising Filter for 3D MR Images
}

\author{
Pierrick Coupé ${ }^{1}$, José V. Manjon², Montserrat Robles², D. Louis Collins ${ }^{1}$. \\ 1 McConnell Brain Imaging Centre, Montréal Neurological Institute, McGill University 3801, \\ University Street, Montréal, Canada H3A 2B4. \\ 2 Biomedical Informatics Group (IBIME), ITACA Institute, Polytechnic University of Valencia, \\ Camino de Vera, s/n. 46022 Valencia, Spain
}

\section{Introduction}

In MR imaging, denoising is an important issue in some clinical uses. Recently a new filter has been introduced by Buades [1]: the Non-Local (NL-) means filter. Based on the natural redundancy of the images, this filter uses comparison of image patches in order to achieve the denoising. Due to its simplicity and high denoising performance, this filter is one of the best state-of-the-art methods $[1,2]$. Nevertheless, according to the nature of the underlying structures the filtering parameters should be adapted. To implicitly optimize the amount of denoising according to the underlying structure, an adaptive multiresolution framework is proposed.

\section{Method}

By combining in the wavelet domain the results obtained with different sets of filtering parameters, the denoising can be improved [3]. We propose to automatically adapt the mixing procedure according to the noise level and the spatial information contained in the detailed subbands.

The original image $I$ is denoised with two sets of filtering parameters: one adapted to the image features preservation and an another adapted to the noise components removal (see [2] and [4] for details). This yields two images: Iu with efficient feature preservation and Io with efficient noise removal (see Fig. 1). After wavelet transformation, the signed distances between absolute values of wavelet coefficients of I and the threshold $\mathrm{T}$ are computed. This signed distance is used to perform a soft mixing between coefficients obtained from the two denoised images (see Fig. 1). When signed distance is superior to zero, the corresponding coefficient is mainly considered as data, thus wavelet coefficient from Iu should be privileged. Inversely, when signed distance is inferior to zero, the corresponding coefficient is mainly considered as noise, thus wavelet coefficient from Io should be privileged. The threshold $\mathrm{T}$ is the BayesSkrink described in [6] and the mixing function a sigmoid.

\section{$\underline{\text { Result }}$}

Synthetic Data: The proposed multiresolution NL-means was tested on the BrainWeb database [5] corrupted with Rician noise at different levels. A comparison between the NL-means $[2,4]$ and the proposed filter was performed by computing Peak Noise Ratio on T1-w phantom. For all the noise levels the proposed multiresolution filter obtained better results (see Fig. 2). 
T1-w data: The proposed method has been applied on a T1-w MR image of 170x256x256 voxels acquired on a 1.5T Philips Gyroscan. Figure 3 shows the denoised image and the residual image.

DTI data with high b-value: The DW-MRI with a $b=3000 \mathrm{~s} / \mathrm{mm} 2$ was composed of 8 B0 images and 100 gradient directions of $63 \times 128 \times 128$ voxels acquired on a 3T Siemens TimeTrio. As proposed in [4] each image and gradient direction has been denoised separately. Figure 4 shows the denoised images.

\section{$\underline{\text { Conclusion }}$}

The results on the BrainWeb MR phantom shows that the proposed Multiresolution NL-means filter outperforms the implementations presented in [2]. Experiments on real data emphasized the potential of the proposed filter on images with high levels of noise such as DW-MRI with high b-values.

\section{$\underline{\text { Reference }}$}

[1] A. Buades, B. Coll, and J.-M. Morel. A review of image denoising algorithms, with a new one. Multiscale Modeling and Simulation, 4(2):490-530, 2005.

[2] P. Coupe, P. Yger, S. Prima, P. Hellier, C. Kervrann, and C. Barillot. An Optimized Blockwise NonLocal Means Denoising Filter for 3-D magnétique Resonance Images. IEEE Transactions on Medical Imaging, 27(4):425-441,

April 2008.

[3] A. Lukin. A multiresolution approach for improving quality of image denoising algorithms. In International Conference on Acoustics, Speech, and Signal Processing (ICASSP'06), pages 857-860, Toulouse, France, May 2006.

[4] N. Wiest-Daessle, S. Prima, P. Coupe, S.P. Morrissey, and C. Barillot. Rician noise removal by non-local means filtering for low signal-to-noise ratio mri: Applications to DT-MRI. In 11th International Conference on Medical Image Computing and Computer-Assisted Intervention, MICCAI'2008, New York, USA, September 2008.

[5] D.L. Collins, A.P. Zijdenbos, V. Kollokian, J.G. Sled, N.J. Kabani, C.J. Holmes, and A.C. Evans. Design and construction of a realistic digital brain phantom. IEEE Transactions on Medical Imaging, 17(3):463-468, 1998.

[6] S. G. Chang, B. Yu, and M. Vetterli. Adaptive wavelet thresholding for image denoising and compression. IEEE Transactions on Image Processing, 9(9):1532- 1546, 2000. 


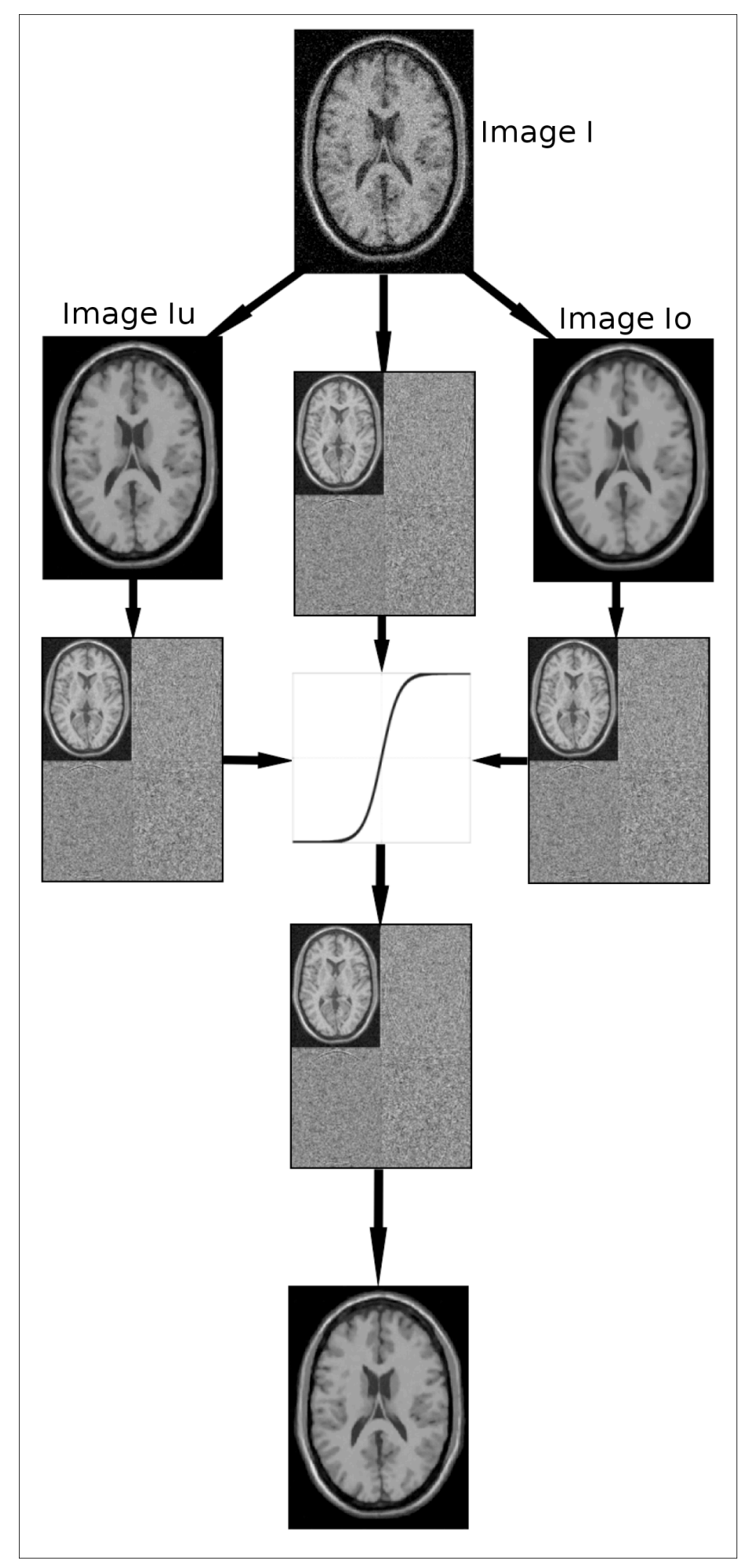

Figure 1. Adaptive Soft Wavelet Coefficient Mixing Procedure. 


\section{T1-w MRI}

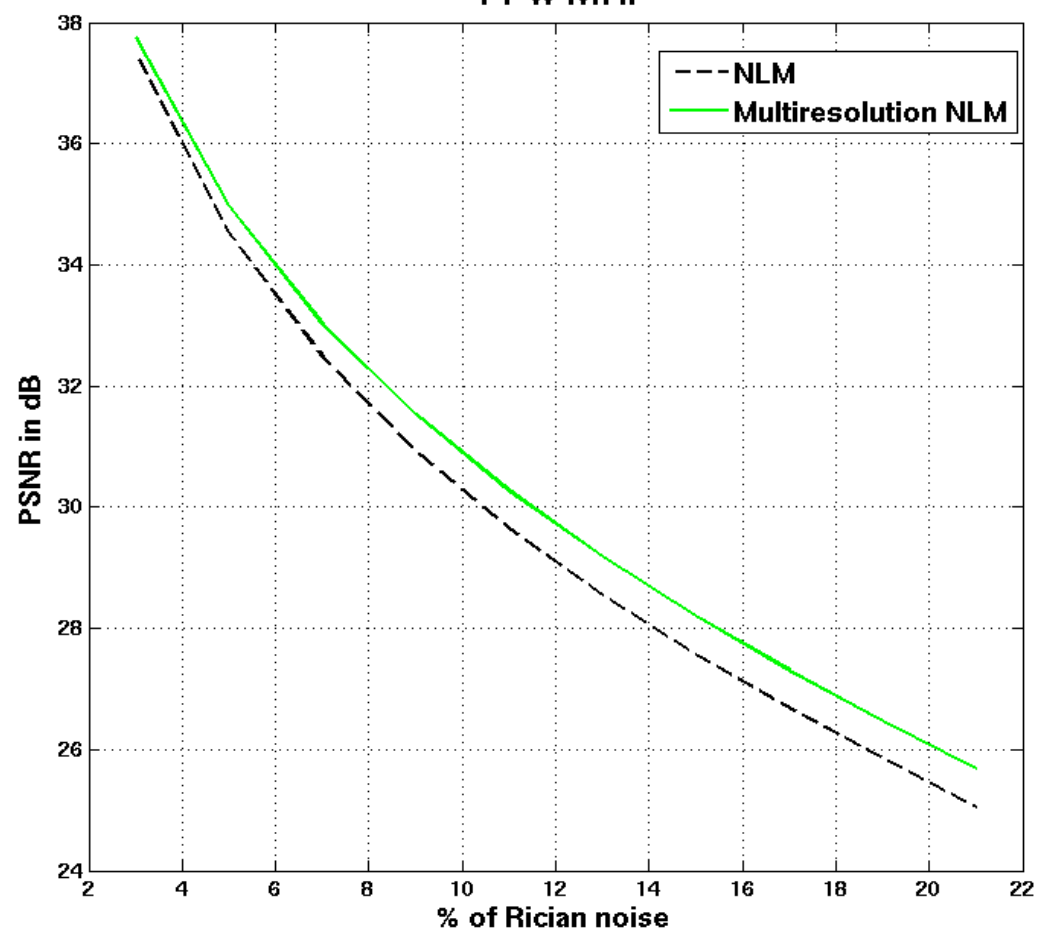

Figure 2. PSNR values of the compared filters on the T1-w phantom for all the levels of Rician noise. In all the cases, the proposed adaptive multiresolution filter obtained the best result. 

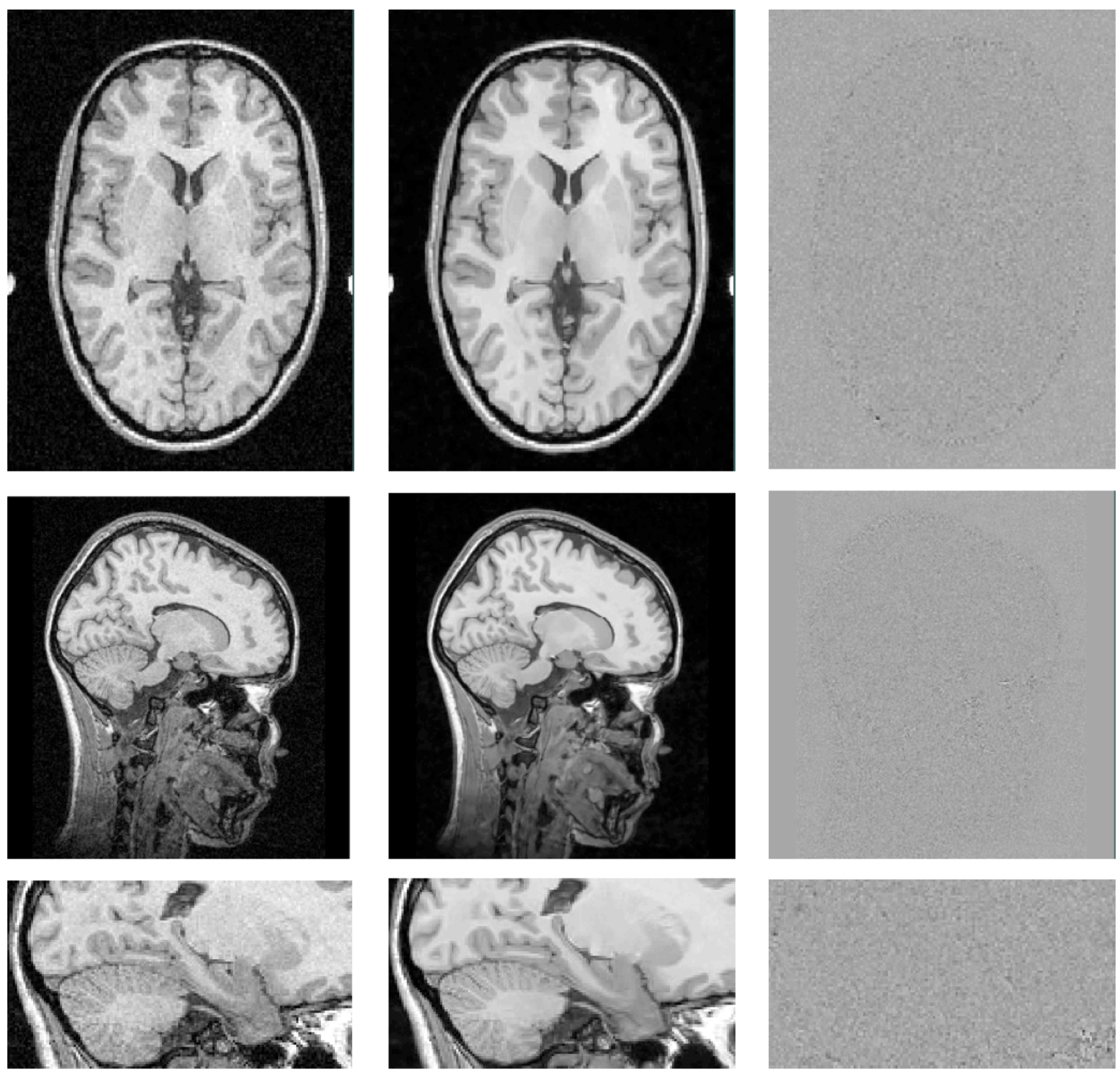

Figure 3. Left: Original T1-w image. Middle: Denoised image obtained with the proposed filter. Right: Residual image (i.e. removed noise). 

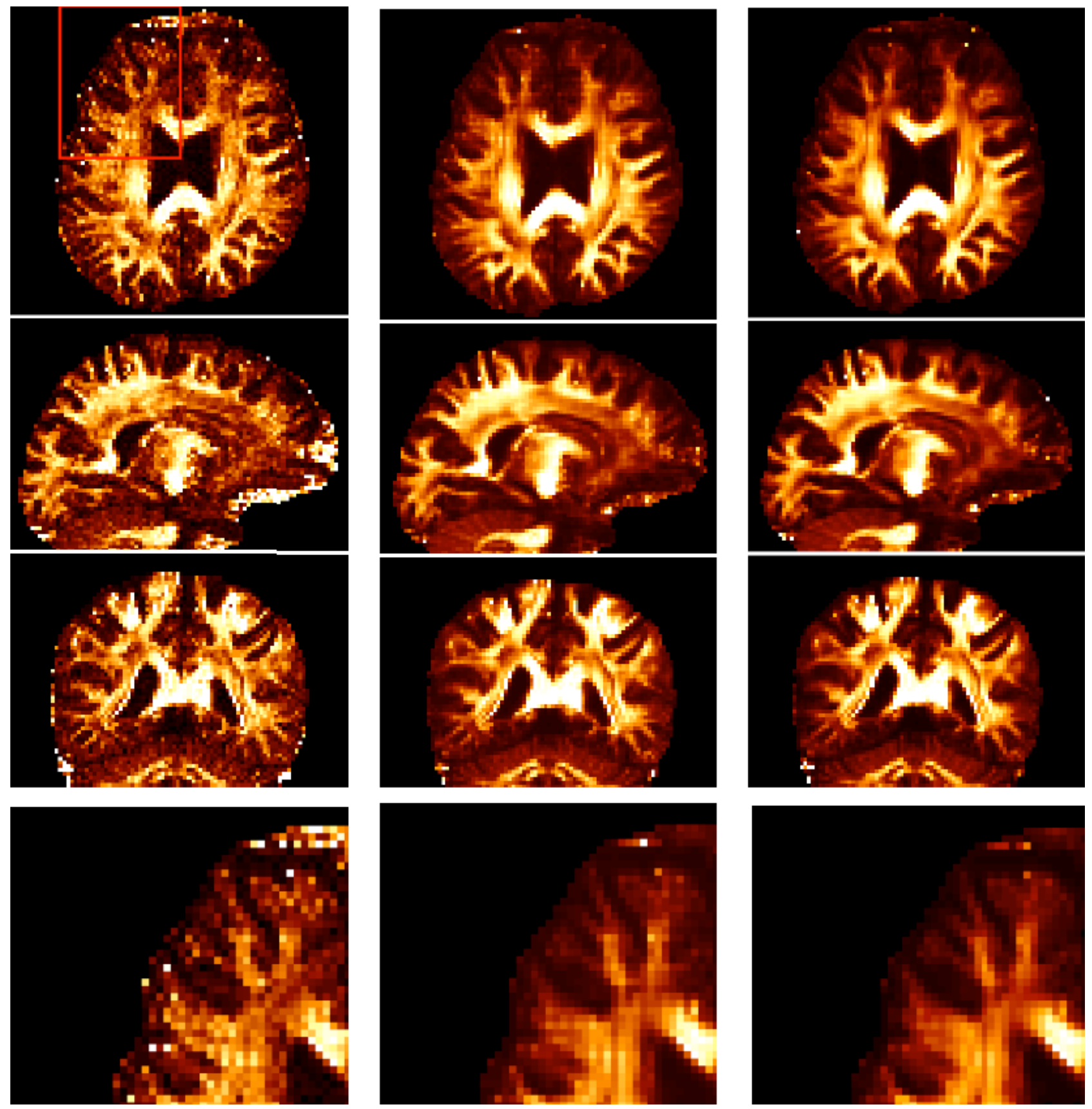

Figure 4. Fractional Anisotropy (FA) map computed on the DW-MRI dataset with high b-value. Left: FA map computed with noisy DW-MRI. Middle: FA map computed on denoised DW-MRI with NLM filter. Right: FA map computed on denoised DW-MRI with the proposed filter. 